\title{
LQG Control Over Lossy TCP-like Networks With Probabilistic Packet Acknowledgements
}

\author{
E. Garone, B. Sinopoli and A. Casavola
}

\begin{abstract}
This paper is concerned with control applications over lossy data networks. Sensor data is transmitted to an estimation-control unit over a network, and control commands are issued to subsystems over the same network. Sensor, control and acknowledgement packets may be randomly lost according to a Bernoulli process. In this context, the discrete-time Linear Quadratic Gaussian (LQG) optimal control problem is considered. We can show how the partial loss of acknowledgements makes the optimal control law a nonlinear function of the information set. For the special case of complete state observation we can compute the optimal controller and show that the stability range increases monotonically with the arrival rate of the acknowledgement packets.
\end{abstract}

\section{INTRODUCTION}

This paper is concerned with the problem of the design and analysis of control systems when components are connected via packet based communication networks. This requires a generalization of classical control techniques that explicitly takes into account the stochastic nature of the communication channel.

In our analysis, we distinguish between two classes of protocols. The distinction resides simply in the availability of packet acknowledgements. Adopting the framework proposed by Imer et al. [1], we will refer therefore to TCP-like protocols if packet acknowledgements are available and to UDP-like protocols otherwise.

We consider a generalized formulation of the Linear Quadratic Gaussian (LQG) optimal control problem by modeling the arrival of both observations and control packets as random processes whose parameters are related to the characteristics of the communication channel. Accordingly, two independent Bernoulli processes are considered, with parameters $\bar{\gamma}$ and $\bar{\nu}$, that govern packet losses between the sensors and the estimation-control unit, and between the latter and the actuation points. Furthermore we introduce a third Bernoulli process of parameter, $\bar{\theta}$, which models the loss of the acknowledgement packet. The goal of this paper is to provide some partial answers to the question of how control

This research was supported in part by CyLab at Carnegie Mellon under grant DAAD19-02-1-0389 from the Army Research Office. Foundation. The views and conclusions contained here are those of the authors and should not be interpreted as necessarily representing the official policies or endorsements, either express or implied, of ARO, CMU, or the U.S. Government or any of its agencies.

E. Garone and A. Casavola are with the Dipartimento di Elettronica, Informatica e Sistemistica, Università degli Studi della Calabria, Via Pietro Bucci, Cubo 42-c, Rende (CS), 87036, Italy \{egarone, casavola\}edeis.unical.it

B. Sinopoli is with the Department of Electrical and Computer Engineering, Carnegie Mellon University, Pittsburgh, PA 15213, USA brunoseece.cmu.edu

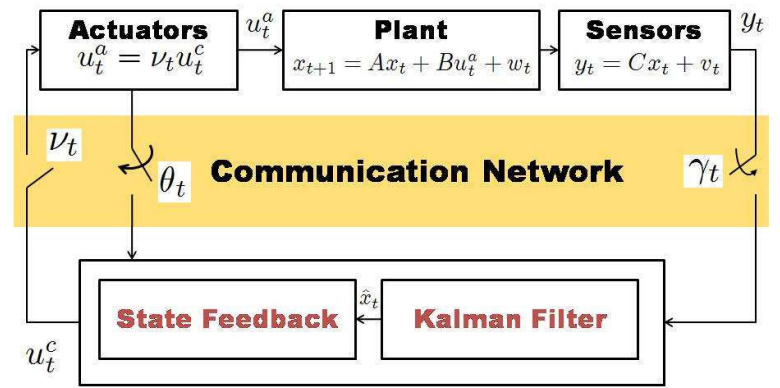

Fig. 1. Overview of the system. Architecture of the closed loop system over a communication network. The binary random variables $\nu_{t}, \gamma_{t}$ and $\theta_{t}$ indicates whether packets are transmitted successfully.

loop performance is affected by communication constraints and what are the basic system-theoretic implications of using unreliable networks for control.

We have shown in some previous work [2], [3], [4] the existence of a critical domain of values for the parameters of the Bernoulli arrival processes, $\bar{\nu}$ and $\bar{\gamma}$, outside which a transition to instability occurs and the optimal controller fails to stabilize the system. In particular, we have shown that under TCP-like protocols the critical arrival probabilities for the control and observation channels are independent of each other. A more involved situation regards UDP-like protocols. In this case the critical arrival probabilities for the control and observation channels are coupled. The stability domain and the performance of the optimal controller degrade considerably as compared with TCP-like protocols as shown in Figure 2.

We have also shown that in the TCP-like case the classic separation principle holds, and consequently the controller and estimator can be designed independently. Moreover, the optimal controller is a linear function of the state. In sharp contrast, in the UDP-like case, the optimal controller is in general non-linear. In this case the absence of an acknowledgement structure generates a nonclassical information pattern [5]. Because of the importance of UDP protocols for wireless sensor networks, we have analyzed a special case when the arrival of a sensor packet provides complete knowledge of the state, despite the lack of acknowledgements, the optimal control design problem yields a linear controller [3]. Also, for the general case, a sub-optimal 
solution was provided in [6], by designing the optimal linear static regulator, composed by constant gains for both the observer and the controller. This is particularly attractive for sensor networks, where simplicity of implementation is highly desirable and complexity issues are a primary concern. In this paper, we drop the assumption of deterministic and instantaneous available of acknowledgement. Loss of acknowledgement leads once again to a nonclassical information pattern, and we are able to prove that in general the optimal control law is a nonlinear function of the information set. By restricting ourselves to the complete observability case, we are able to solve the LQG problem. We show that probabilistic acknowledgements increase the stability range of the system. Furthermore we can show how such range converges to the TCP-like one as the erasure probability for the acknowledgement channel tends to zero.

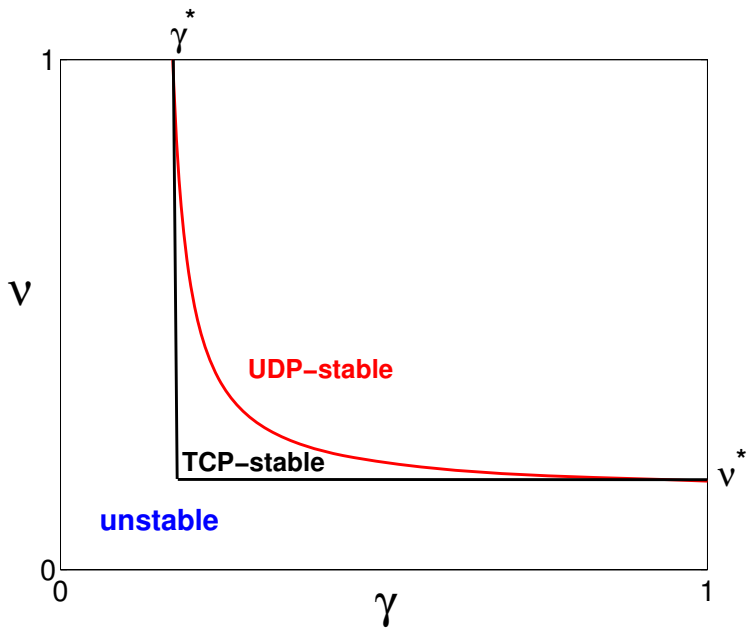

Fig. 2. Region of stability for UDP-like and TCP-like optimal control relative to measurement packet arrival probability $\bar{\gamma}$, and the control packet arrival probability $\bar{\nu}$.

In the past few years networked embedded control systems have drawn considerable attention in the academic world. We will now try to set our work in the context of the existing literature. In [7] and [8], an estimator, i.e. a Kalman filter, is placed at the sensor side of the link and no assumption is made on the statistical model of the data loss process. Smith et al. [9] focused on designing a suboptimal yet computationally efficient estimator for Markov Chain arrival processes. In [10] the authors study the stability of Kalman Filter under general Markovian packet losses. In [11], the authors present a simple estimation scheme that is able to recover the fate of the control packet under UDP-like protocols by constraining the control signal sent to the plant. Drew et al [12] analyze the problem of designing a controller over a wireless LAN. Control design has been investigated in the context of Cross Layer Design by Liu et al [13]. Finally, in [14],[15] the plant and the controller are modeled as deterministic time invariant discrete-time systems connected to zero-mean stochastic structured uncertainty, where the variance of the stochastic perturbation is a function of the Bernoulli parameters. Here, the controller design is posed an an optimization problem to maximize mean-square stability of the closed loop system. While this method allows analysis of Multiple Input Multiple Output (MIMO) systems with many different controller and receiver compensation schemes [14], it does not include process and observation noise. The resulting controller is restricted to be time-invariant, hence sub-optimal. Finally, within the context of UDP-like control, Epstein et al. [11] recently proposed to estimate not only the state of the system, but also a binary variable which indicates wether the previous control packet has been received or not. Such strategy, improves closed loop performance at the price of a somewhat larger computational complexity.

The remainder of this paper is organized as follows. Section 2 provides the problem formulation. In Section 3 we derive the estimator equations. In section 4 we consider the control problem in the general case. Section 5 considers the special case of complete observability. Section 6 provides conclusions and directions for future work. To make the paper more readable we moved all the proofs to the Appendix contains all the proofs.

\section{Problem Statement and Formulation}

Consider the following linear stochastic system with intermittent observation and control packets:

$$
\begin{aligned}
& x_{k+1}=A x_{k}+B u_{k}^{a}+\omega_{k} \\
& u_{k}^{a}=\nu_{k} u_{k}^{c}+\left[1-\nu_{k}\right] u_{k}^{l} \\
& y(k)=\gamma_{k} C x_{k}+v_{k}
\end{aligned}
$$

where $u_{k}^{a}$ is the control input to the actuator, $u_{k}^{c}$ is the desired control input computed by the controller, $\left(x_{0}, \omega_{k}, v_{k}\right)$ are Gaussian, uncorrelated, white, with mean $\left(\overline{x_{0}}, 0,0\right)$ and covariance $\left(P_{0}, Q, R\right)$ respectively, and $\gamma_{k}$ and $\nu_{k}$ are i.i.d. Bernoulli random variable with $P\left(\gamma_{k}=1\right)=\overline{\gamma_{k}}$ and $P\left(\gamma_{k}=1\right)=\overline{\gamma_{k}} \cdot u_{k}^{l}$ is the signal it is locally provided to the actuators in the case $\nu_{k}=0$ (all packet to the actuators are lost).

It is possible to choose $u_{k}^{l}$ in several way, the principal strategy are:

1) zero-input scheme $u_{k}^{l}=0$

2) hold-input scheme $u_{k}^{l}=u_{k}^{c}$

It is important to define which is the Information Set the controller handle with. In previous publications ([1],), usually two kind of protocols are discussed:

$$
I_{k}= \begin{cases}F_{k}=\left\{\gamma_{k} y_{k}, \gamma_{k}, \nu_{k-1} \mid k=0, \ldots, t\right\} & \text { TCP }- \text { like } \\ G_{k}=\left\{\gamma_{k} y_{k}, \gamma_{k} \mid k=0, \ldots, t\right\} & \text { UDP - like }\end{cases}
$$

It is a common experience in wireless "open-air" networks the fact that using "secure" TCP-like protocols can have a very bad effect on the communication bandwidth, due to the 
big amount of packet collisions. Otherwise UDP-like protocols based Kalman filter exhibit much lower performances with respect to TCP protocols.

A possible way to deal with such a problem is to use an intermediate solution, that is to send a single message command and a single acknowledgement packet through UDP-like channels. This means that during each process we have a certain non-zero probability $(1-\bar{\theta})$ to not receive any acknowledgment by the actuator.

$$
E_{k}=\left\{\gamma_{k} y_{k}, \gamma_{k}, \theta_{k-1}, \theta_{k-1} v_{k-1} \mid k=0, \ldots, t\right\}
$$

Moreover consider the following cost function

$$
\begin{aligned}
& J_{N}\left(u^{N-1}, \overline{x_{0}}, P_{0}\right)= \\
& E\left[x_{N}^{T} W_{N} x_{N}+\sum_{k=0}^{N-1} x_{k}^{T} W_{k} x_{k}+u_{k}^{a T} U_{k} u_{k}^{a} \mid u^{N-1}, \overline{x_{0}}, P_{0}\right] .
\end{aligned}
$$

where $u^{N-1}=u_{N-1}, u_{N-2}, \ldots, u_{1}$. The aim here is, given at each time instant the the information set $E_{k}$, to compute the optimal control input sequence $u^{*}(\cdot)=$ $g(k, I(k))$ such that it minimize the functional (2) i.e.: $\min _{u_{k}=g_{k}\left(I_{k}\right)} J_{N}\left(u^{N-1}, \overline{x_{0}}, P_{0}\right)$.

\section{ESTIMATOR DESIGN}

Let's consider the system

$$
\begin{aligned}
& x_{k+1}=A x_{k}+\nu_{k} B u_{k}+\omega_{k} \\
& y_{k}=\gamma_{k} C x_{k}+v_{k}
\end{aligned}
$$

And let be $\theta_{k}$ the probability to receive the acknowledgment. The one predition of the Kalman filter becomes

$$
\begin{aligned}
& \hat{x}_{k+1 \mid k}=A \hat{x}_{k \mid k}+\theta_{k} \nu_{k} B u_{k}+\left(1-\theta_{k}\right) \bar{\nu} B u_{k} \\
& e_{k+1 \mid k}=x_{k+1}-\hat{x}_{k+1 \mid k} \\
& \hat{x}_{k+1 \mid k}=A \hat{x}_{k \mid k}+\theta_{k} \nu_{k} B u_{k}+\left(1-\theta_{k}\right) \bar{\nu} B u_{k} \\
& e_{k+1 \mid k}=x_{k+1}-\hat{x}_{k+1 \mid k}=A x_{k}+\nu_{k} B u_{k}+\omega_{k} \\
& -A \hat{x}_{k \mid k}+\theta_{k} \nu_{k} B u_{k}-\left(1-\theta_{k}\right) \bar{\nu} B u_{k}= \\
& =A e_{k \mid k}+\left(\nu_{k}-\theta_{k} \nu_{k}-\left(1-\theta_{k}\right) \bar{\nu}\right) B u_{k}+\omega_{k}
\end{aligned}
$$

Then the covariance is:

$$
\begin{aligned}
& P_{k+1 \mid k}=E\left[e_{k+1 \mid k} e_{k+1 \mid k}^{T} \mid E_{k}, \theta_{k}, \theta_{k} \nu_{k}\right]= \\
& =E\left[A e_{k \mid k} e_{k \mid k}^{T} A \mid E_{k}\right]+E\left[\omega_{k} \omega_{k}^{T} \mid E_{k}\right] \\
& +E\left[\left(\nu_{k}-\theta_{k} \nu_{k}-\left(1-\theta_{k}\right) \bar{\nu}\right)^{2} \mid E_{k}, \theta_{k}, \theta_{k} \nu_{k}\right] B u_{k} u_{k}^{T} B^{T} \\
& P_{k+1 \mid k}=A P_{k \mid k} A^{T}+Q \\
& +E\left[\left(\nu_{k}-\theta_{k} \nu_{k}-\left(1-\theta_{k}\right) \bar{\nu}\right)^{2} \mid E_{k}, \theta_{k}, \theta_{k} \nu_{k}\right] B u_{k} u_{k}^{T} B^{T}
\end{aligned}
$$

Finally we get

$$
P_{k+1 \mid k}=A P_{k \mid k} A^{T}+Q+\left(1-\theta_{k}\right)(1-\bar{\nu}) \bar{\nu}\left[B u_{k} u_{k}^{T} B^{T}\right]
$$

The correction step instead remains the one showed in [16]:

$$
\begin{aligned}
& \hat{x}_{k+1 \mid k+1}=\hat{x}_{k+1 \mid k}+\gamma_{k+1} K_{k+1}\left(y_{k+1}-C x_{k+1 \mid k}\right) \\
& P_{k+1 \mid k+1}=P_{k+1 \mid k}-\gamma_{k+1} K_{k+1} C P_{k+1 \mid k} \\
& K_{k+1}=P_{k+1 \mid k} C^{T}\left(C P_{k+1 \mid k} C^{T}+R\right)^{-1}
\end{aligned}
$$

Remark 1: Note that:

$\theta_{k}=1 \Rightarrow \quad P_{k+1 \mid k}=A P_{k \mid k} A+Q$

$\theta_{k}=0 \Rightarrow \quad P_{k+1 \mid k}=A P_{k \mid k} A+Q+\bar{\nu}(1-\bar{\nu})\left[B u_{k} u_{k}^{T} B^{T}\right]$

this means that, at each time $k$, the prediction switch between the "TCP-style" predictions or the UDP ones, depending on the instant value of $\theta_{k}$.

\section{Optimal Control - General CASE}

Here we show that, in the general case, the optimal control law is not a linear function of the state estimate and that, the estimation and control design cannot be treated separately.

To prove that let us consider the following very simple case: let $\mathrm{A}=\mathrm{B}=\mathrm{C}=\mathrm{W}_{N}=\mathrm{W}_{k}=\mathrm{R}=1, \mathrm{U}_{k}=\mathrm{Q}=0$.

Let us define

$$
V(N)=E\left[x_{N}^{T} W_{N} x_{N} \mid E_{N}\right]=E\left[x_{N}^{2} \mid E_{N}\right]
$$

for $k=N-1$ we will have:

$$
\begin{aligned}
& V_{N-1}\left(x_{N-1}\right)=\min _{u_{N}} E\left[x_{N-1}^{2}+V_{N}\left(x_{N}\right) \mid E_{N-1}\right]= \\
& \min _{u_{N}} E\left[x_{N-1}^{2}+x_{N}^{2} \mid E_{N-1}\right] \\
& \quad=\min _{u_{N}} E\left[x_{N-1}^{2}+\left(x_{N-1}+\nu_{N-1} u_{N-1}\right)^{2} \mid E_{N-1}\right]= \\
& E\left[2 x_{N-1 \mid N-1}^{2} \mid E_{N-1}\right]+\min _{u_{N}} \bar{\nu}\left(u_{N-1}^{2}+2 \hat{x}_{N-1 \mid N-1} u_{N-1}\right)
\end{aligned}
$$

The optimal input is then:

$$
u_{N-1}=-\hat{x}_{N-1 \mid N-\hat{1}}
$$

Then, if we substitute back this solution in (3) the cost becomes

$$
\begin{aligned}
& V_{N-1}(x)=E\left[2 x_{N-1}^{2} \mid E_{N-1}\right]-\bar{\nu} \hat{x}_{N-1 \mid N-1}^{2}= \\
& (2-\bar{\nu}) E\left[x_{N-1}^{2} \mid G\right]-\bar{\nu} P_{N-1 \mid N-1}
\end{aligned}
$$

Let us focus on the covariance matrix:

$$
\begin{aligned}
& P_{N-1 \mid N-1}=P_{N-1 \mid N-2}-\gamma_{N-1} \frac{P_{N-1 \mid N-2}^{2}}{\left(P_{N-1 \mid N-2}+1\right)}= \\
& =P_{N-1 \mid N-2}-\gamma_{N-1}\left(P_{N-1 \mid N-2}^{-} 1+\frac{1}{\left(P_{N-1 \mid N-2}+1\right)}\right)
\end{aligned}
$$

Becouse of

$$
P_{N-1 \mid N-2}=P_{N-2 \mid N-2}+\left(1-\theta_{N-2}\right)(1-\bar{\nu}) \bar{\nu} u_{N-2}^{2}
$$

Then:

$$
\begin{aligned}
& E\left[P_{N-1 \mid N-1} \mid E_{N-2}\right]=P_{N-2 \mid N-2}+(1-\bar{\theta})(1-\bar{\nu}) \bar{\nu} u_{N-2}^{2} \\
& -\bar{\gamma}\left(P_{N-2 \mid N-2}+(1-\bar{\theta})(1-\bar{\nu}) \bar{\nu} u_{N-2}^{2}-1+\right. \\
& \left.\bar{\theta} \frac{1}{P_{N-2 \mid N-2}}+(1-\bar{\theta}) \frac{1}{P_{N-2 \mid N-2}+(1-\bar{\nu}) \bar{\nu} u_{N-2}^{2}}\right)
\end{aligned}
$$

Finally we get

$$
\begin{aligned}
& V_{N-2}(x)=\min _{u_{N-2}} E\left[x_{N-2}^{2}+V_{N-1}\left(x_{N-1}\right) \mid E_{N-2}\right]= \\
& (3-\bar{\nu}) E\left[x_{N-1}^{2} \mid E_{N-2}\right]+\min _{u_{N-2}} P_{N-2 \mid N-2}+ \\
& (1-\bar{\theta})(1-\bar{\nu}) \bar{\nu} u_{N-2}^{2}-\bar{\gamma}\left(P_{N-2 \mid N-2}+\right. \\
& (1-\bar{\theta})(1-\bar{\nu}) \bar{\nu} u_{N-2}^{2}-1+\bar{\theta} \frac{1}{P_{N-2 \mid N-2}} \\
& \left.+(1-\bar{\theta}) \frac{1}{P_{N-2 \mid N-2}+(1-\bar{\nu}) \bar{\nu} u_{N-2}^{2}}\right)^{-}
\end{aligned}
$$


The first terms within the last parenthesis are convex quadratic functions of the control input $u_{N-2}$, however the last term is not. Therefore, the optimal control law is, in general, a nonlinear function of the information set $E_{k}$. Such a nonlinearity arises from the fact that the correction error covariance matrix $P_{k+1 \mid k+1}$ is a non-linear function of the innovation error covariance $P_{k+1 \mid k}$.

Theorem 1: Let us consider the stochastic system defined in Equations 81) with horizon $N \geq 2$. Then:

- Unless $\bar{\theta}=1$ (TCP-like case), the separation principle does not hold

- The optimal control feedback $u_{k}=g_{k}^{*}\left(E_{k}\right)$ that minimizes the cost functional defined in Equation (2) is, in general, a nonlinear function of information set $E_{k}$

- The optimal control feedback $u_{k}=g_{k}^{*}\left(E_{k}\right)$ is a linear function of the estimated state if and only if one of the following conditions holds true:

- $\bar{\theta}=1$

- $C$ is invertible $R=0$

in such a case, in the infinite horizon scenario, if it exist the optimal state-feedback gain is constant, i.e. $L_{k}^{*}=$ $L^{*}$, and can be computed as the solution of a convex optimization problem.

Proof. It follows by inspection.

\section{Optimal Control $-\mathrm{C}$ invertible, $\mathrm{R}=0$ Case}

Without loss of generality we can assume $C=I$ (it is always possible to use a linear transformation $z=C x$ ). Because of the hypothesis that there is no measurement noise, i.e. $R=0$, it is possible to simply measure the state $x_{k}$ when a packet is delivered. The estimator equations simplify as follow:

$$
\begin{aligned}
& K_{k+1}=I \\
& P_{k+1 \mid k}=A P_{k \mid k} A+Q+\left(1-\theta_{k}\right)(1-\bar{\nu}) \bar{\nu}\left[B u_{k} u_{k}^{T} B^{T}\right] \\
& P_{k+1 \mid k+1}=\left(1-\gamma_{k+1}\right) P_{k+1 \mid k}= \\
& \left(1-\gamma_{k+1}\right)\left(A P_{k \mid k} A+Q+\left(1-\theta_{k}\right)(1-\bar{\nu}) \bar{\nu}\left[B u_{k} u_{k}^{T} B^{T}\right]\right) \\
& E\left[P_{k+1 \mid k+1} \mid E_{k}\right]= \\
& (1-\bar{\gamma})\left(A P_{k \mid k} A+Q+(1-\bar{\theta})(1-\bar{\nu}) \bar{\nu}\left[B u_{k} u_{k}^{T} B^{T}\right]\right)
\end{aligned}
$$

In the last equation the independence of $E_{k}, \gamma_{k+1}, \theta_{k}$ are exploited.

Following the classical dynamic programming approach to optimal control, we claim that the value function $V_{k}^{*}\left(x_{k}\right)$ can be written as follows:

$$
\begin{aligned}
& V_{k}\left(x_{k}\right)=\hat{x}_{k \mid k}^{T} S_{k} \hat{x}_{k \mid k}+\operatorname{trace}\left(T_{k} P_{k \mid k}\right)+\operatorname{trace}\left(D_{k} Q\right)= \\
& E\left[x_{k \mid k}^{T} S_{k} x_{k \mid k}\right]+\operatorname{trace}\left(H_{k} P_{k \mid k}\right)+\operatorname{trace}\left(D_{k} Q\right)
\end{aligned}
$$

For each $k=N, \ldots, 0$ where $H_{k}=T_{k}-S_{k}$.

This is clearly true for $k=N$, in fact we have:

$$
\begin{aligned}
& V_{N}\left(x_{N}\right)=E\left[x_{N}^{T} W_{N} x_{N} \mid E_{N}\right]= \\
& \hat{x}_{N \mid N}^{T} W_{N} \hat{x}_{N \mid N}+\operatorname{trace}\left(W_{N} P_{N \mid N}\right)
\end{aligned}
$$

therefore the statement is satisfied by $S_{N}=T_{N}=$ $W_{N}, D_{N}=0$.

Let us suppose that Equation (5)is true for $k+1$ and let us show by induction it holds true for $k$ :

$$
\begin{aligned}
& V_{k}\left(x_{k}\right)=\min _{u_{k}} E\left[x_{k}^{T} W_{k} x_{k}+\nu_{k} u_{k}^{T} U_{k} u_{k}+V_{k+1}\left(x_{k+1}\right) \mid E_{k}\right]= \\
& \min _{u_{k}} E\left[x_{k}^{T} W_{k} x_{k} E_{k}\right]+\bar{\nu} u_{k}^{T} U_{k} u_{k}+E\left[x_{k+1}^{T} S_{k+1} x_{k+1} \mid E_{k}\right]+ \\
& \operatorname{trace}\left(H_{k+1} P_{k+1 \mid k+1}\right)+\operatorname{trace}\left(D_{k+1} Q\right)= \\
& \min _{u_{k}} E\left[x_{k}^{T} W_{k} x_{k} \mid E_{k}\right]+\bar{\nu}_{k} u_{k}^{T} U_{k} u_{k}+ \\
& +E\left[\begin{array}{c}
\left(A x_{k \mid k}+\theta_{k} \nu_{k} B u_{k}+\left(1-\theta_{k}\right) \bar{\nu} B u_{k}\right)^{T} S_{k+1} \mid \\
\left(A x_{k \mid k}+\theta_{k} \nu_{k} B u_{k}+\left(1-\theta_{k}\right) \bar{\nu} B u_{k}\right)
\end{array}\right]+ \\
& +\operatorname{trace}\left(H_{k+1}\left((1-\bar{\gamma})\left(A P_{k \mid k} A+Q+\left(1-\theta_{k}\right) \bar{\nu}(1-\bar{\nu})\left[B u_{k} u_{k}^{T} B^{T}\right]\right)\right)\right) \\
& +\operatorname{trace}\left(D_{k+1} Q\right)
\end{aligned}
$$

Then it becomes:

$$
\begin{aligned}
& V_{k}\left(x_{k}\right)=\min _{u_{k}} E\left[x_{k}^{T} W_{k} x_{k}+\bar{\nu}_{k} u_{k}^{T} U_{k} u_{k}+\right. \\
& \left.\left(x_{k \mid k}^{T} A^{T} S_{k+1} A x_{k \mid k}\right)+\left(\theta_{k} \nu_{k} u_{k}^{T} B^{T} B u_{k}\right)+\left(1-\theta_{k}\right) \bar{\nu} u_{k}^{T} B^{T} B u_{k}^{T}\right)+ \\
& \left.2 \theta_{k} \nu_{k} x_{k \mid k}^{T} A^{T} S_{k+1} B u_{k}+2\left(1-\theta_{k}\right) \bar{\nu} x_{k \mid k}^{T} A^{T} S_{k+1} B u_{k} \mid E_{k}\right]+ \\
& \quad \operatorname{trace}\left(H_{k+1}\left((1-\bar{\gamma})\left(A P_{k \mid k} A+Q+(1-\bar{\theta}) \bar{\nu}(1-\bar{\nu})\left[B u_{k} u_{k}^{T} B^{T}\right]\right)\right)\right) \\
& +\operatorname{trace}\left(D_{k+1} Q\right)=E\left[x_{k \mid k}^{T}\left(W_{k}+A^{T} S_{k+1} A\right) x_{k \mid k}\right]+ \\
& (1-\bar{\gamma}) \operatorname{trace}\left(H_{k+1}\left(\left(A P_{k \mid k} A+Q\right)\right)\right)+\operatorname{trace}\left(D_{k+1} Q\right) \\
& +\min _{u_{k}} \bar{\nu}\left(u_{k}^{T}\left(U_{k}+B^{T}\left(S_{k+1}+(1-\bar{\theta})(1-\bar{\nu}) \bar{\nu} H_{k+1}\right) B\right) u_{k}^{T}\right)+ \\
& 2 \bar{\nu}\left(x_{k \mid k}^{T} A^{T} S_{k+1} B u_{k}\right)
\end{aligned}
$$

Since $V_{k}\left(x_{k}\right)$ is a convex quadratic function w.r.t. $u_{k}$, the minimizer is the solution

of $\partial V_{k}\left(x_{k}\right) / \partial u_{k}=0$ which is given by:

$$
\begin{aligned}
& u_{k}^{*}=-\left(U_{k}+B^{T}\left(S_{k+1}+\bar{\alpha} H_{k+1}\right) B\right)^{-1}\left(B^{T} S_{k+1} A x_{k \mid k}\right) \\
& =L_{k} x_{k \mid k}
\end{aligned}
$$

where $\bar{\alpha}=(1-\bar{\gamma})(1-\bar{\theta})(1-\bar{\nu}) \bar{\nu}$. which is linear function of the estimated state $x_{k \mid k}$. Substituting back into the value function we get:

$$
\begin{aligned}
& V_{k}\left(x_{k}\right)=\operatorname{trace}\left((1-\bar{\gamma}) H_{k+1}\left(\left(A P_{k \mid k} A\right)\right)\right) \\
& + \text { trace }\left(\left((1-\bar{\gamma}) T_{k+1}+D_{k+1}\right) Q\right) \\
& +E\left[x_{k \mid k}^{T}\left(W_{k}+A^{T} S_{k+1} A\right) x_{k \mid k}\right]-\bar{\nu} x_{k \mid k}^{T}\left(A^{T} S_{k+1} B L_{k}\right) x_{k \mid k}
\end{aligned}
$$

It becomes

$V_{k}\left(x_{k}\right)=\operatorname{trace}\left((1-\bar{\gamma}) H_{k+1}\left(\left(A P_{k \mid k} A\right)\right)\right)+$ $E\left[x_{k \mid k}^{T}\left(W_{k}+A^{T} S_{k+1} A\right) x_{k \mid k}+\left(\bar{\nu}\left(x_{k \mid k}^{T} A^{T} S_{k+1} B\right) L_{k} x_{k \mid k}\right)\right]+$ $\operatorname{trace}\left(\left(D_{k+1}+(1-\bar{\gamma}) T_{k+1}\right) Q\right)-\operatorname{trace}\left(\left(\bar{\nu} A^{T} S_{k+1} B L_{k} P_{k \mid k}\right)\right)$

Then finally we obtain

$V_{k}\left(x_{k}\right)=\operatorname{trace}\left(\left(D_{k+1}+(1-\bar{\gamma}) H_{k+1}\right) Q\right)+$ $E\left[x_{k \mid k}^{T}\left(W_{k}+A^{T} S_{k+1} A\right) x_{k \mid k}+\left(\bar{\nu}\left(x_{k \mid k}^{T} A^{T} S_{k+1} B\right) L_{k} x_{k \mid k}\right)\right]+$ + trace $\left(\left(\left((1-\bar{\gamma}) A^{T} H_{k+1} A-\bar{\nu} A^{T} S_{k+1} B L_{k}\right) P_{k \mid k}\right)\right)$

From the last equation we see that the value function can be written as in Equation (5) if and only if the following equations are satisfied:

$$
S_{k}=W_{k}+A^{T} S_{k+1} A .+\bar{\nu}\left(A^{T} S_{k+1} B\right) L_{k}
$$




$$
\begin{gathered}
T_{k}=(1-\bar{\gamma}) A^{T} T_{k+1} A+W_{k}+\bar{\gamma} A^{T} S_{k+1} A \\
D_{k}=D_{k+1}+(1-\bar{\gamma}) T_{k+1}+\bar{\gamma} S_{k+1}
\end{gathered}
$$

Remark 2: Notice that, if $\bar{\theta} \rightarrow 0$, the UDP special case presented is [16] is reached.

The optimal minimal cost for the finite horizon, $J_{N}^{*}=$ $V_{0}\left(x_{0}\right)$ is then given by: For the infinite horizon optimal controller, necessary and sufficient conditions for the average minimal cost $J_{\infty}^{*}=\lim _{N \rightarrow \infty} J_{N}^{*}$ to be finite, are that the coupled iterative Equations (7) and (6) should converge to a finite value $S_{\infty}$ and $T_{\infty}$ as $N \rightarrow \infty$.

Theorem 2: Consider the system (1) and consider the problem of minimizing the cost function (2) within the class of admissible policies $u_{k}=f\left(E_{k}\right)$.Assume also that $R=0$ and $C$ is square and invertible. Then:

1) The optimal estimator gain is constant and in particular $K_{k}=I$ if $C=I$.

2) The infinite horizon optimal control exists if and only if there exists positive definite matrices $S_{\infty}, T_{\infty}>0$ such that $S_{\infty}=\Phi_{S}\left(S_{\infty}, T_{\infty}\right)$ and $T_{\infty}=\Phi_{T}\left(S_{\infty}, T_{\infty}\right)$, where $\Phi_{S}$ and $\Phi_{T}$ are:

$$
\begin{gathered}
\Phi_{S}\left(S_{k}, W_{k}\right)=W_{k}+A^{T} S_{k} A-\bar{\nu}\left(A^{T} S_{k} B\right) \\
\left(U_{k}+B^{T}\left((1-\bar{\alpha}) S_{k+1}+\bar{\alpha} T_{k+1}\right) B\right)^{-1}\left(B^{T} S_{k+1} A\right) \\
\Phi_{T}\left(S_{k}, T_{k}\right)=(1-\bar{\gamma}) A^{T} T_{k+1} A+W_{k}+\bar{\gamma} A^{T} S_{k+1} A
\end{gathered}
$$

3) The infinite horizon optimal controller gain is constant: $\lim _{k \rightarrow \infty} L_{k}=L_{\infty}$

$$
L_{\infty}=-\left(U+B^{T}\left((1-\bar{\alpha}) S_{\infty}+\bar{\alpha} T_{\infty}\right) B\right)^{-1}\left(B^{T} S_{\infty} A\right)
$$

4) A necessary condition for existence of $S_{\infty}, T_{\infty}>0$ is

$$
\begin{aligned}
& 1-|A|^{2}\left(1-\frac{\bar{\nu}}{(1-\bar{\alpha})+\bar{\alpha} \frac{\gamma|A| 2}{1-(1-\gamma)|A|^{2}}}\right) \geq 0 \\
& \bar{\gamma}>1-\frac{1}{|A|^{2}}
\end{aligned}
$$

where $|A|=\max _{i}\left|\lambda_{i}(A)\right|$ is the largest eigenvalue of the matrix $A$. This condition is also sufficient if $B$ is square and invertible.

5) The expected minimum cost for the infinite horizon scenario converges to:

$$
J_{\infty}^{*}=\lim _{k \rightarrow \infty} \frac{1}{N} J_{N}^{*}=\operatorname{trace}\left(\left((1-\bar{\gamma}) T_{k}+\bar{\gamma} S_{k}\right) Q\right)
$$

Proof: 1) This fact follows from Equations (4). Statements 2), 3) and 5) follow from Lemma 2 (See Appendix) and Equations (6) and (7). Statement 5) corresponds to Lemmas 3 and 4 (See Appendix).

\section{CONCLUSiONS}

In this paper we analyzed a generalized version of the LQG control problem in the case where both observation and control packets may be lost during transmission over a communication channel. This situation arises frequently in distributed systems where sensors, controllers and actuators

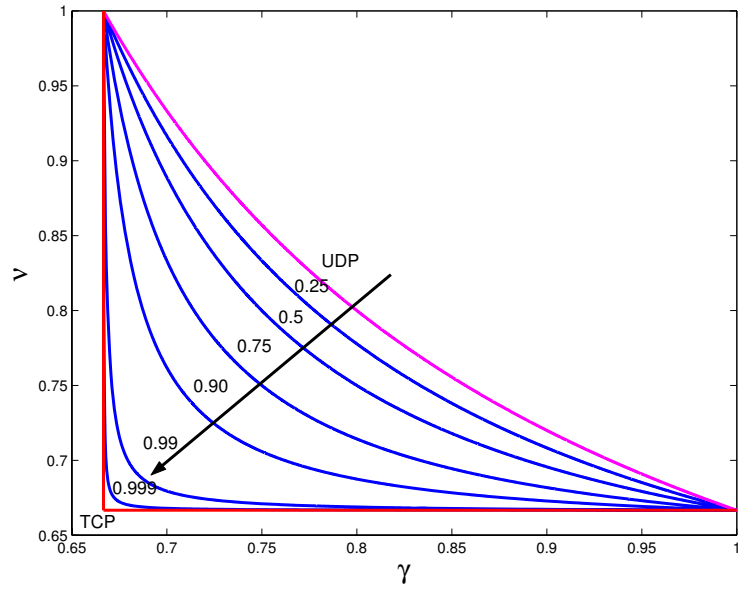

Fig. 3. Region of stability relative to measurement packet arrival probability $\bar{\gamma}$, and the control packet arrival probability $\bar{\nu}$, parametrized into the acknowledgment packet arrival probability $\bar{\theta}$

reside in different physical locations and have to rely on data networks to exchange information. In this context controller design heavily depends on the communication protocol used. In fact, in TCP-like protocols, acknowledgements of successful transmissions of control packets are provided to the controller, while in UDP-like protocols, no such feedback is provided. In the first case, the separation principle holds and the optimal control is a linear function of the state. As a consequence, controller and estimator design problems are decoupled. UDP-like protocols present a much more complex problem. We have shown that the even partial lack of acknowledgement of control packets results in the failure of the separation principle. Estimation and control are now intimately coupled. We have shown that the LQG optimal control is, in general, nonlinear in the estimated state. In the particular case, where we have access to full state information, the optimal controller is linear in the state. In this particular case we could show how the partial presence of acknowledgement increases the stability range of the overall system, converging to the TCP-like with deterministic acknowledgements as the arrival rate for the acknowledgement packets tends to one.

\section{APPENDIX: PROOFS}

Lemma 1: Let $S, T \in \mathbb{M}=\left\{M \in \mathbb{R}^{n \times n} \mid M \geq 0\right\}$. Consider the operators $\Phi^{S}(S, T)$, and $\Phi^{T}(S, T)$ as defined in Equations (6) and (7), and consider the sequences $S_{k+1}=\Phi^{S}\left(S_{k}, T_{k}\right)$ and $T_{k+1}=\Phi^{T}\left(S_{k}, T_{k}\right)$. Consider $L_{S, T}^{*}=-\left(U+B^{\prime}((1-\bar{\alpha}) S+\bar{\alpha} T) B\right)^{-1} B^{\prime} S A$ and the operator:

$$
\begin{aligned}
& \Upsilon(S, T, L)=\left(1-\frac{\bar{\nu}}{1-\bar{\alpha}}\right) A^{\prime} S A+W+ \\
& \quad+\frac{\bar{\nu}}{1-\bar{\alpha}}(A+(1-\bar{\alpha}) B L)^{\prime} S(A+(1-\bar{\alpha}) B L)+ \\
& \quad+\bar{\nu} L^{\prime} U L+\bar{\nu} \bar{\alpha} L^{\prime} B^{\prime} T B L
\end{aligned}
$$

Then the following facts are true: 
(a) $\Phi^{S}(S, T)=\min _{L} \Upsilon(S, T, L)$

(b) $0 \leq \Upsilon\left(S, T, L_{S, T}^{*}\right)=\Phi^{S}(S, T) \leq \Upsilon(S, T, L) \forall L$

(c) If $S_{k+1}>S_{k}$ and $T_{k+1}>T_{k}$, then $S_{k+2}>S_{k+1}$ and $T_{k+2}>T_{k+1}$.

(d) If the pair $\left(A, W^{1 / 2}\right)$ is observable and $S=$ $\Phi^{S}(S, T)$ and $T=\Phi^{T}(S, T)$, then $S>0$ and $T>0$.

Proof:

(a) If $U$ is invertible then it is easy to verify by direct substitution substitution that

$$
\begin{aligned}
\Upsilon(S, T, L)= & \Phi^{S}(S, T)+ \\
& +\bar{\nu}\left(L-L_{S, T}^{*}\right)^{\prime}\left(U+B^{\prime}((1-\bar{\alpha}) S+\bar{\alpha} T) B\right)\left(L-L_{S, T}^{*}\right) \\
\geq & \Phi^{S}(S, T)
\end{aligned}
$$

(b) The nonnegativeness follows form the observation that $\Upsilon(S, T, L)$ a sum of positive semi-definite matrices. In fact $\left(1-\frac{\bar{\nu}}{1-\bar{\alpha}}\right) \geq 0$ and $0 \leq \bar{\alpha} \leq 1$. The equality $\Upsilon\left(S, T, L_{S, T}^{*}\right)=\Phi^{S}(S, T)$ can be verified by direct substitution. The last inequality follows directly from Fact (b).

(c)

$$
\begin{aligned}
S_{k+2} & =\Phi^{S}\left(S_{k+1}, T_{k+1}\right)=\Upsilon\left(S_{k+1}, T_{k+1}, L_{S_{k+1}, T_{k+1}}^{*}\right) \\
& \geq \Upsilon\left(S_{k}, T_{k}, L_{S_{k+1}}^{*}, T_{k+1}\right) \geq \Upsilon\left(S_{k}, T_{k}, L_{S_{k}, T_{k}}^{*}\right) \\
& =\Phi^{S}\left(S_{k}, T_{k}\right)=S_{k+1} \\
T_{k+2} & =\Phi^{T}\left(S_{k+1}, T_{k+1}\right) \geq \Phi^{T}\left(S_{k}, T_{k}\right)=T_{k+1}
\end{aligned}
$$

(d) First observe that $S=\Phi^{S}(S, T) \geq 0$ and $T=\Phi^{T}(S, T) \geq 0$. Thus, to prove that $S, T>0$, we only need to establish that $S, T$ are nonsingular. Suppose they are singular, the there exist vectors $0 \neq v_{s} \in \mathcal{N}(S)$ and $0 \neq v_{t} \in \mathcal{N}(T)$, i.e. $S v_{s}=0$ and $T v_{t}=0$, where $\mathcal{N}(\cdot)$ indicates the null space. Then

$$
\begin{aligned}
0 & =v_{s}^{\prime} S v_{s}=v_{s}^{\prime} \Phi^{S}(S, T) v_{s}=v_{s}^{\prime} \Upsilon\left(S, T, L_{S, T}^{*}\right) v_{s} \\
& =\left(1-\frac{\bar{\nu}}{1-\bar{\alpha}}\right) v_{s}^{\prime} A^{\prime} S A v_{s}+v_{s}^{\prime} W v_{s}+\star
\end{aligned}
$$

where $\star$ indicates other terms. Since all the terms are positive semi-definite matrices, this implies that all the term must be zero:

$$
\begin{aligned}
& v_{s}^{\prime} A^{\prime} S A v_{s}=0 \Longrightarrow S A v_{s}=0 \Longrightarrow A v_{s} \in \mathcal{N}(S) \\
& v_{s}^{\prime} W v_{s}=0 \Longrightarrow W^{1 / 2} v_{s}=0
\end{aligned}
$$

As a result, the null space $\mathcal{N}(S)$ is $A$-invariant. Therefore, $\mathcal{N}(S)$ contains an eigenvector of $A$, i.e. there exists $u \neq 0$ such that $S u=0$ and $A u=\sigma u$. As before, we conclude that $W u=0$. This implies (using the PBH test) that the pair $\left(A, W^{1 / 2}\right)$ is not observable, contradicting the hypothesis. Thus, $\mathcal{N}(S)$ is empty, proving that $S>0$. The same argument can be used to prove that also $T>0$.

Lemma 2: Consider the following operator:

$$
\begin{aligned}
\Upsilon(S, T, L)= & A^{\prime} S A+W+2 \bar{\nu} A^{\prime} S B L+ \\
& +\bar{\nu} L^{\prime}\left(U+B^{\prime}((1-\bar{\alpha}) S+\bar{\alpha} T) B\right) L
\end{aligned}
$$

Assume that the pairs $\left(A, W^{1 / 2}\right)$ and $(A, B)$ are observable and controllable, respectively. Then the following statements are equivalent: (a) There exist a matrix $\tilde{L}$ and positive definite matrices $\tilde{S}$ and $\tilde{T}$ such that:

$$
\tilde{S}>0, \tilde{T}>0, \tilde{S}=\Upsilon(\tilde{S}, \tilde{T}, \tilde{L}), \tilde{T}=\Phi^{T}(\tilde{S}, \tilde{T})
$$

(b) Consider the sequences:

$$
S_{k+1}=\Phi^{S}\left(S_{k}, T_{k}\right), \quad T_{k+1}=\Phi^{T}\left(S_{k}, T_{k}\right)
$$

where the operators $\Phi^{S}(\cdot), \Phi^{T}(\cdot)$ are defined in Equations (6) and (7). For any initial condition $S_{0}, T_{0} \geq 0$ we have

$$
\lim _{k \rightarrow \infty} S_{k}=S_{\infty}, \quad \lim _{k \rightarrow \infty} T_{k}=T_{\infty}
$$

and $S_{\infty}, T_{\infty}>0$ are the unique positive definite solution of the following equations

$$
S_{\infty}=\Phi^{S}\left(S_{\infty}, T_{\infty}\right), \quad T_{\infty}=\Phi^{T}\left(S_{\infty}, T_{\infty}\right)
$$

Proof: See [16].

Lemma 3: Let us consider the fixed points of Equations (6) and (7), i.e. $S=\Phi^{S}(S, T), T=\Phi^{T}(S, T)$ where $S, T \geq$ 0 . Let $A$ be unstable. A necessary condition for existence of solution is

$$
\begin{aligned}
& 1-|A|^{2}\left(1-\frac{\bar{\nu}}{\left.(1-\bar{\alpha})+\bar{\alpha} \frac{\gamma|A| 2 \mid}{1-(1-\gamma)|A|^{2}}\right) \geq 0}\right. \\
& \bar{\gamma}>1-\frac{1}{|A|^{2}}
\end{aligned}
$$

where $|A| \triangleq \max _{i}\left|\lambda_{i}(A)\right|$ is the largest eigenvalue of the matrix $A$.

Proof: To prove the necessity condition it is sufficient to show that there exist some initial conditions $S_{0}, T_{0} \geq 0$ for which the sequences $S_{k+1}=$ $\Phi^{S}\left(S_{k}, T_{k}\right), T_{k+1}=\Phi^{T}\left(S_{k}, T_{k}\right)$ are unbounded, i.e. $\lim _{k \rightarrow \infty} S_{k}=\lim _{k \rightarrow \infty} T_{k}=\infty$. To do so, suppose that at some time-step $k$ we have $S_{k} \geq s_{k} v v^{\prime}$ and $T_{k} \geq t_{k} v v^{\prime}$, where $s_{k}, t_{k}>0$, and $v$ is the eigenvector corresponding to the largest eigenvalue of $A^{\prime}$, i.e. $A^{\prime} v=\lambda_{\max } v$ and $\left|\lambda_{\max }\right|=\left|A^{\prime}\right|=|A|$. Then we have:

$$
\begin{aligned}
S_{k+1}= & \Phi^{S}\left(S_{k}, T_{k}\right) \geq \Phi^{S}\left(s_{k} v v^{\prime}, t_{k} v v^{\prime}\right) \\
= & \min _{L} \Upsilon\left(s_{k} v v^{\prime}, t_{k} v v^{\prime}, L\right) \\
= & \min _{L}\left(s_{k} A^{\prime} v v^{\prime} A+W+2 s_{k} \bar{\nu} A^{\prime} v v^{\prime} B L+\right. \\
& \left.\quad+\bar{\nu} L^{\prime}\left(U+B^{\prime}\left((1-\bar{\alpha}) s_{k} v v^{\prime}+\bar{\alpha} t_{k} v v^{\prime}\right) B\right) L\right) \\
\geq & \min _{L}\left(s_{k}|A|^{2} v v^{\prime}+2 s_{k} \bar{\nu} \lambda_{\max } v v^{\prime} B L+\right. \\
& \left.\quad+\bar{\nu} L^{\prime} B^{\prime}\left((1-\bar{\alpha}) s_{k} v v^{\prime}+\bar{\alpha} t_{k} v v^{\prime}\right) B L\right) \\
= & \min _{L}\left(s_{k}|A|^{2} v v^{\prime}-\frac{|A|^{2} \bar{\nu} s_{k}^{2}}{\xi_{k}} v v^{\prime}+\right. \\
& \left.\quad+\bar{\nu} \xi_{k}\left(\lambda_{\max } s_{k}^{2} I+\frac{1}{\xi_{k}} B L\right)^{\prime} v v^{\prime}\left(\lambda_{\max } s_{k}^{2} I+\frac{1}{\xi_{k}} B L\right)\right) \\
\geq & s_{k}|A|^{2} v v^{\prime}-\frac{|A|^{2} \bar{\nu} s_{k}^{2}}{(1-\bar{\alpha}) s_{k}+\bar{\alpha} t_{k}} v v^{\prime} \\
= & |A|^{2} s_{k}\left(1-\frac{\bar{\nu} s_{k}}{(1-\bar{\alpha}) s_{k}+\bar{\alpha} t_{k}}\right) v v^{\prime} \\
= & s_{k+1} v v^{\prime}
\end{aligned}
$$


where $I$ is the identity matrix and $\xi_{k}=(1-\bar{\alpha}) s_{k}+\bar{\alpha} t_{k}$. Similarly we have:

$$
\begin{aligned}
T_{k+1} & =\Phi^{T}\left(S_{k}, T_{k}\right) \geq \Phi^{T}\left(s_{k} v v^{\prime}, t_{k} v v^{\prime}\right) \\
& =(1-\bar{\gamma}) t_{k} A^{\prime} v v^{\prime} A+\bar{\gamma} s_{k} A^{\prime} v v^{\prime} A+W \\
& \geq(1-\bar{\gamma}) t_{k}\left|A^{2}\right| v v^{\prime}+\bar{\gamma} s_{k}|A|^{2} v v^{\prime} \\
& \left.=|A|^{2}\left((1-\bar{\gamma}) t_{k}+\bar{\gamma} s_{k}\right)\right) v v^{\prime} \\
& =t_{k+1} v v^{\prime}
\end{aligned}
$$

We can summarize the previous results as follows:

$$
\begin{gathered}
\left(S_{k} \geq s_{k} v v^{\prime}, T_{k} \geq t_{k} v v^{\prime}\right) \Rightarrow \\
\Rightarrow\left(S_{k+1} \geq s_{k+1} v v^{\prime}, T_{k+1} \geq t_{k+1} v v^{\prime}\right) \\
s_{k+1}=\phi^{s}\left(s_{k}, t_{k}\right)=|A|^{2} s_{k}\left(1-\frac{\bar{\nu} s_{k}}{(1-\bar{\alpha}) s_{k}+\bar{\alpha} t_{k}}\right), \\
\left.t_{k+1}=\phi^{t}\left(s_{k}, t_{k}\right)=|A|^{2}\left((1-\bar{\gamma}) t_{k}+\bar{\gamma} s_{k}\right)\right)
\end{gathered}
$$

Let us define the following sequences:

$$
\begin{array}{cl}
S_{k+1}=\Phi^{S}\left(S_{k}, T_{k}\right), & T_{k+1}=\Phi^{T}\left(S_{k}, T_{k}\right), \quad S_{0}=T_{0}=v v^{\prime} \\
s_{k+1}=\phi^{s}\left(s_{k}, t_{k}\right), & t_{k+1}=\phi^{t}\left(s_{k}, t_{k}\right), \quad s_{0}=t_{0}=1 \\
\tilde{S}_{k}=s_{k} v v^{\prime}, & \tilde{T}_{k}=t_{k} v v^{\prime}
\end{array}
$$

From the previous derivations we have that $S_{k} \geq \tilde{S}_{k}, T_{k} \geq$ $\tilde{T}_{k}$ for all time $k$. Therefore, it is sufficient to find when the scalar sequences $s_{k}, t_{k}$ diverges to find the necessary conditions. It should be evident that also the operators $\phi^{s}(s, t), \phi^{t}(s, t)$ are monotonic in their arguments. Also it should be evident that the only fixed points of $s=$ $\phi^{s}(s, t), t=\phi^{t}(s, t)$ are $s=t=0$. Therefore we should be find when the origin is an unstable equilibrium point, since in this case $\lim _{k \rightarrow \infty} s_{k}, t_{k}=\infty$. Note that $t=\phi^{t}(s, t)$ can be written as:

$$
\begin{aligned}
t & =\Phi^{T}(s, t)=(1-\bar{\gamma})|A|^{2} t+\bar{\gamma}|A|^{2} s \\
& =\psi(s)=\frac{\bar{\gamma}|A|^{2} s}{1-(1-\bar{\gamma})|A|^{2}}
\end{aligned}
$$

with the additional constraint $1-(1-\bar{\gamma}) A^{2}>0$. A necessary condition for stability for the origin is that the origin of restricted map $z_{k+1}=\phi\left(z_{k}, \psi\left(z_{k}\right)\right)$ is stable. The restricted map is given by:

$$
\begin{aligned}
z_{k+1} & =|A|^{2} z_{k}\left(1-\bar{\nu} \frac{z_{k}}{(1-\bar{\alpha}) z_{k}+\bar{\alpha} \frac{\bar{\gamma}|A|^{2}}{1-(1-\bar{\gamma}) A^{2}} z_{k}}\right) \\
& =|A|^{2}\left(1-\frac{\bar{\nu}}{(1-\bar{\alpha})+\bar{\alpha} \frac{\bar{\gamma}|A|^{2}}{1-(1-\bar{\gamma}) A^{2}}}\right) z_{k} .
\end{aligned}
$$

This is a linear map and it is stable only if the term inside the parenthesis is smaller than unity, i.e.

$$
1-|A|^{2}\left(1-\frac{\bar{\nu}}{(1-\bar{\alpha})+\bar{\alpha} \frac{\gamma|A| 2}{1-(1-\gamma)|A|^{2}}}\right)<1
$$

which concludes the lemma.
Lemma 4: Let us consider the fixed points of Equations (6) and (7), i.e. $S=\Phi^{S}(S, T), T=\Phi^{T}(S, T)$ where $S, T \geq$ 0 . Let $A$ be unstable, $\left(A, W^{1 / 2}\right)$ observable and $B$ square and invertible. Then a sufficient condition for existence of solution is

$$
\begin{aligned}
& 1-|A|^{2}\left(1-\frac{\bar{\nu}}{(1-\bar{\alpha})+\bar{\alpha} \frac{\gamma|A| 2}{1-(1-\gamma)|A|^{2}}}\right)<1 \\
& \bar{\gamma}>1-\frac{1}{|A|^{2}}
\end{aligned}
$$

where $|A| \triangleq \max _{i}\left|\lambda_{i}(A)\right|$ is the largest eigenvalue of the matrix $A$.

Proof: The proof is constructive. In fact we find a control feedback gain $\tilde{L}$ that satisfies the conditions stated in Theorem 2(a). Let $\tilde{L}=-\eta B^{-1} A$ where $\eta>0$ is a positive scalar that is to be determined. Also consider $S=s I, T=$ $t I$, where $I$ is the identity matrix and $s, t>0$ are positive scalars. Then we have

$$
\begin{aligned}
\Upsilon(s I, t I, \tilde{L})= & A^{\prime} s A+W-2 \bar{\nu} \eta A^{\prime} s A+\bar{\nu} A^{\prime} B^{-^{\prime}} U B^{-1} A+ \\
& +\bar{\nu} \eta^{2} A^{\prime}((1-\bar{\alpha}) s+\bar{\alpha} t) A \\
\leq & |A|^{2}\left(s-2 \bar{\nu} s \eta+\bar{\nu}((1-\bar{\alpha}) s+\bar{\alpha} t) \eta^{2}\right) I+w I \\
= & \varphi^{s}(s, t, \eta) I \\
= & \bar{\gamma} A^{\prime} s A+(1-\bar{\gamma}) A^{\prime} t A+W \\
\leq & \left(\bar{\gamma}|A|^{2} s+(1-\bar{\gamma})|A|^{2} t\right) I+w I \\
\leq & \varphi^{t}(s, t) I
\end{aligned}
$$

where $w=\left|W+\bar{\nu} A^{\prime} B^{-^{\prime}} U B^{-1} A\right|>0$ and $I$ is the identity matrix. Let us consider the following scalar operators and sequences:

$$
\begin{aligned}
\varphi^{s}(s, t, \eta) & =|A|^{2}\left(1-2 \bar{\nu} \eta+\bar{\nu}(1-\bar{\alpha}) \eta^{2}\right) s+\bar{\nu} \bar{\alpha} \eta^{2} t+w \\
\varphi^{t}(s, t) & =\bar{\gamma}|A|^{2} s+(1-\bar{\gamma})|A|^{2} t+w \\
s_{k+1} & =\varphi^{s}\left(s_{k}, t_{k}, \eta\right), \quad t_{k+1}=\varphi^{t}\left(s_{k}, t_{k}\right), \quad s_{0}=t_{0}=0
\end{aligned}
$$

The operators are clearly monotonically increasing in $s, t$, and since $s_{1}=\varphi^{s}\left(s_{0}, t_{0}, \eta\right)=w \geq s_{0}$ and $t_{1}=\varphi^{t}\left(s_{0}, t_{0}\right)=$ $w \geq t_{0}$, it follows that the sequences $s_{k}, t_{k}$ are monotonically increasing. If these sequences are bounded, then they must converge to $\tilde{s}, \tilde{t}$. Therefore $s_{k}, t_{k}$ are bounded if and only if there exist $\tilde{s}, \tilde{t}>0$ such that $\tilde{s}=\varphi^{s}(\tilde{s}, \tilde{t}, \eta)$ and $\tilde{t}=\varphi^{t}(\tilde{s}, \tilde{t})$. Let us find the fixed points:

$$
\begin{aligned}
& \tilde{t}=\varphi^{t}(\tilde{s}, \tilde{t}) \Rightarrow \\
& \tilde{t}=\frac{\bar{\gamma}|A|^{2}}{1-(1-\bar{\gamma})|A|^{2}} \tilde{s}+w_{t}
\end{aligned}
$$

where $w_{t} \triangleq \frac{w}{1-(1-\bar{\gamma})|A|^{2}}>0$, and we must have $1-(1-$ $\bar{\gamma})|A|^{2}>0$ to guarantee that $\tilde{t}>0$. Substituting back into 
the operator $\varphi^{s}$ we have:

$$
\begin{aligned}
\tilde{s}= & |A|^{2}\left(1-2 \bar{\nu} \eta+\bar{\nu}(1-\bar{\alpha}) \eta^{2}\right) \tilde{s}+\bar{\nu} \bar{\alpha} \eta^{2} \frac{\bar{\gamma}|A|^{2}}{1-(1-\bar{\gamma})|A|^{2}} \tilde{s}+ \\
& +\bar{\nu} \bar{\alpha} \eta^{2} w_{t}+w \\
= & |A|^{2}\left(1-2 \bar{\nu} \eta+\bar{\nu}\left((1-\bar{\alpha})+\frac{\bar{\gamma} \bar{\alpha}|A|^{2}}{1-(1-\bar{\gamma})|A|^{2}}\right) \eta^{2}\right) \tilde{s}+w(\eta) \\
= & a(\eta) \tilde{s}+w(\eta)
\end{aligned}
$$

where $w(\eta) \triangleq \bar{\nu} \bar{\alpha} \eta^{2} w_{t}+w>0$. For a positive solution $\tilde{s}$ to exist, we must have $a(\eta)<1$. Since $a(\eta)$ is a quadratic function of the free parameter $\eta$, we can try to increase the basin of existence of solutions by choosing $\eta^{*}=\operatorname{argmin}_{\eta} a(\eta)$, which can be found by solving $\frac{d a}{d \eta}\left(\eta^{*}\right)=0$ and is given by:

$$
\eta^{*}=\frac{1}{(1-\alpha)+\frac{\gamma \alpha\left|A^{2}\right|}{1-(1-\gamma) A^{2}}}
$$

Therefore a sufficient condition for existence of solutions is given by:

$$
\begin{aligned}
a\left(\eta^{*}\right) & <1 \\
\mid A\left(1-\frac{\nu}{\left((1-\alpha)+\frac{\gamma \alpha|A|^{2}}{1-(1-\gamma) A^{2}}\right)}\right) & <1
\end{aligned}
$$

which is the same bound for the necessary condition of convergence in Lemma 3.

If this condition is satisfied then $\lim _{k \rightarrow \infty} s_{k}=\tilde{s}$ and $\lim _{k \rightarrow \infty} t_{k}=\tilde{t}$. Let us consider now the sequences $\bar{S}_{k}=s_{k} I, \bar{T}_{k}=t_{k} I, S_{k+1}=\Upsilon\left(S_{k}, T_{k}, \tilde{L}\right)$ and $T_{k+1}=$ $\Phi^{T}\left(S_{k}, T_{k}\right)$, where $\tilde{L}=-\eta^{*} B^{-1} A, S_{0}=T_{0}=0$, and $s_{k}, t_{k}$ where defined above. These sequences are all monotonically increasing. From Equations (13) and (14) it follows that $\left(S_{k} \leq s_{k} I, T_{k} \leq t_{k} I\right) \Rightarrow\left(S_{k+1}=\leq s_{k+1} I, T_{k+1} \leq t_{k} I\right)$.

Since this is verified for $k=0$ we can claim that $S_{k}<\tilde{s} I$ and $T_{k}<\tilde{t} I$ for all $k$. Since $S_{k}, T_{k}$ are monotonically increasing and bounded, then they must converge to positive semidefinite matrices $\tilde{S}, \tilde{T} \geq 0$ which solve the equations $\tilde{S}=\Upsilon(\tilde{S}, \tilde{T}, \tilde{L})$ and $\tilde{T}=\bar{\Phi}^{T}(\tilde{S}, \tilde{T})$. Since by hypothesis the pair $\left(A, W^{1 / 2}\right)$ is observable, using similar arguments of Lemma $1(\mathrm{~d})$, it is possible to show that $\tilde{S}, \tilde{T}>0$. Therefore $\tilde{S}, \tilde{T}, \tilde{L}$ satisfy the conditions of Theorem 2(a), from which if follows statement (b) of the same theorem. This implies that the sufficient conditions derived here guarantee the claim of the lemma.

\section{REFERENCES}

[1] O. C. Imer, S. Yuksel, and T. Basar, "Optimal control of dynamical systems over unreliable communication links," in NOLCOS, Stutgart, Germany, 2004.

[2] B. Sinopoli, L. Schenato, M. Franceschetti, K. Poolla, M. Jordan, and S. Sastry, "Optimal control with unreliable communication: the tcp case," in American Control Conference, Portland, OR, June 2005.
[3] B. Sinopoli, L. Schenato, M. Franceschetti, K. Poolla, and S. Sastry, "LQG control with missing observation and control packets," IFAC, 2005.

[4] — , "An LQG optimal linear controller for control systems with packet losses," in Proc. of IEEE CDC, December 2005.

[5] H. Witsenhausen, "A counterexample in stochastic optimum control," SIAM Journal on Control, vol. 6, pp. 131-147, 1968.

[6] B. Sinopoli, L. Schenato, M. Franceschetti, K. Poolla, and S.Sastry, "Optimal linear lqg control over lossy networks without packet acknowledgment," in In Proc. of the 36th Conf. on Decision and Contr., 2006.

[7] V. Gupta, D. Spanos, B. Hassibi, and R. M. Murray, "Optimal LQG control across a packet-dropping link," Submitted to Systems and Control Letters, 2005.

[8] Y. Xu and J. Hespanha, "Estimation under controlled and uncontrolled communications in networked control systems," in To be presented at the IEEE Conference on Decision and Control, Sevilla, Spain, December 2005.

[9] S. Smith and P. Seiler, "Estimation with lossy measuraments: jump estimators for jump systems," IEEE Transaction on Automatic Control, vol. 48, no. 12, pp. 1453-1464, 2003.

[10] M. Huang and S. Dey, "Stability of kalman filtering with markovian packet losses," Automatica, To appear.

[11] M. Epstein, L. Shi, and R. M. Murray, "An estimation algorithm for a class of networked control systems using udp-like communication schemes," in IEEE Conf. on Decision and Control, 2006.

[12] M. Drew, X. Liu, A. Goldsmith, and J. Hedrick, "Networked control system design over a wirelss lan," in Proceedings of the IEEE CDC, 2005.

[13] X. Liu and A. J. Goldsmith, Cross-layer Design of Distributed Control over Wireless Networks, T. Basar, Ed. Birkhauser (In Press), 2005.

[14] N. Elia and J. Eisembeis, "Limitation of linear control over packet drop networks," in Proceedings of IEEE Conference on Decision and Control, vol. 5, Bahamas, December 2004, pp. 5152-5157.

[15] N. Elia, "Remote stabilization over fading channels," Systems and Control Letters, vol. 54, pp. 237-249, 2005.

[16] L. Schenato, B. Sinopoli, M. Franceschetti, K. Poolla, and S. Sastry, "Foundations of control and estimation over lossy networks," Proc. of the IEEE, February 2007, to appear. 